\title{
Electrical and Sensing Properties of Polyaniline /Molydbenumtrioxide composites
}

\author{
Sweta M Nayak ${ }^{1}$, Enugandula Sravani $^{2}{ }^{, N_{a r s a p p a} \text { Reddy }^{3}, \text { Jakeer Husain }^{4 *} \text {,Jaisheel sagar }}{ }^{5}$ \\ Department of Physics, Gulbarga University Kalaburagi, Karnataka ${ }^{1}$ \\ Assistant Professor Dept of Physics Princeton degree \& PG college Osmania university Hyderabad ${ }^{2}$ \\ Associate Professor ECM Department JBIET Moinabad . Hydrabad, India ${ }^{3}$ \\ Assistant Professor Dept of Engg Physics Faculty of Engineering and Technology, KBNU Gulbarga, \\ Karnataka ,India.585104 \\ Assistant Professor Dept of Mechanical Engineering Lord's Institute of Engg And Technology Hyderabad ${ }^{5}$ \\ *Author of Correspondence: Dr.Jakeer Husain
}

\begin{abstract}
MoO}_{3}$ Polyaniline nanocomposites were synthesized by in-situ polymerization technique. The formation of nanocomposites was characterized by scanning electron microscopy (SEM) The SEM image shows a substantial change in morphology after incorporating nano particle and the presence of nano particle in composites was noticed. The electrical and Sensing properties of these composite are studied at room temperature by using two probe method.
\end{abstract}

Keywords:SEM,PANI,Composite Conductivity.

\section{INTRODUCTION}

Polyaniline is the most widely studied because of its ease to synthesis, low density, less cost, soluble in various solvents, good processibility Polyanilne has also been used in many applications like microwave absorption devices and rechargeable battery [1]. Recently, the demand for various kinds of microwave absorbers has increased in the frequency range of $1-20 \mathrm{GHz}$, because of their twofold use, EMI shielding and countermeasure to radar detection. Suppression of EMI and meeting the electromagnetic compatibility (EMC) have become an essential requirement in industries dealing with high-speed wireless data communication systems, [2-4]. Application of appropriate microwave absorbing material in electronic equipments, help to control the excessive self-emission of electromagnetic waves and ensures the undisturbed functioning of the equipment in the presence of external electromagnetic interference. [5]. Application of microwave absorbing coatings on the exterior surfaces of military aircraft and vehicles helps to avoid detection by the radar [6-10].

\section{EXPERIMENTAL}

\subsection{SYNTHESIS OF POLYANILINE.}

Aniline hydrochloride (equi molar volume of aniline and hydrochloride acid) was dissolved in distilled water in a volumetric flask to $100 \mathrm{ml}$ of solution. Ammoniumpersulphate $(0.25 \mathrm{M})$ was dissolved in water also to $100 \mathrm{ml}$ of solution.Both solutions were kept for 1 hour at room temperature, then mixed in a beaker, stirred with a mechanical stirrer, and left at rest to polymerize. Next day, the PANI precipitate was collected on a filter, washed with $0.2 \mathrm{M} \mathrm{HCL}$, and similarly with acetone.

\subsection{Preparation of Polyaniline/ $\mathrm{MoO}_{3}$ composites}

Aniline $(0.1 \mathrm{M})$ was mixed in $1 \mathrm{M} \mathrm{HCl}$ and stirred for $15 \mathrm{~min}$ to form aniline hydrochloride. $\mathrm{MoO}_{3}$ nanoparticles were added in the mass fraction to the above solution with vigorous stirring in order to keep the $\mathrm{MoO}_{3}$ homogeneously suspended in the solution. To this solution, $0.1 \mathrm{M}$ of ammonium persulphate, which acts as an oxidizer was slowly added drop-wise with continuous stirring at $5{ }^{\circ} \mathrm{C}$ for $4 \mathrm{~h}$ to completely polymerize. The precipitate was filtered, washed with deionized water, Acetone, and finally dried in an oven for $24 \mathrm{~h}$ to achieve a constant mass.

\subsection{Scanning electron microscopy}

\section{RESULT AND DISCUSSION}

The particles exhibit a compact arrangement of homogeneous roughly spherical in shape. Most of the particles are found to be grouped. fig.3.1 (b) shows that the Polyaniline/ $\mathrm{MoO}_{3}$ composites are cluster like structure and the presence of such sharp crystal of $\mathrm{MoO}_{3}$ has a strong influence on various electrical parameters of these composites 


\section{International Advanced Research Journal in Science, Engineering and Technology}

Vol. 8, Issue 6, June 2021

DOI: $10.17148 / I A R J S E T .2021 .8674$

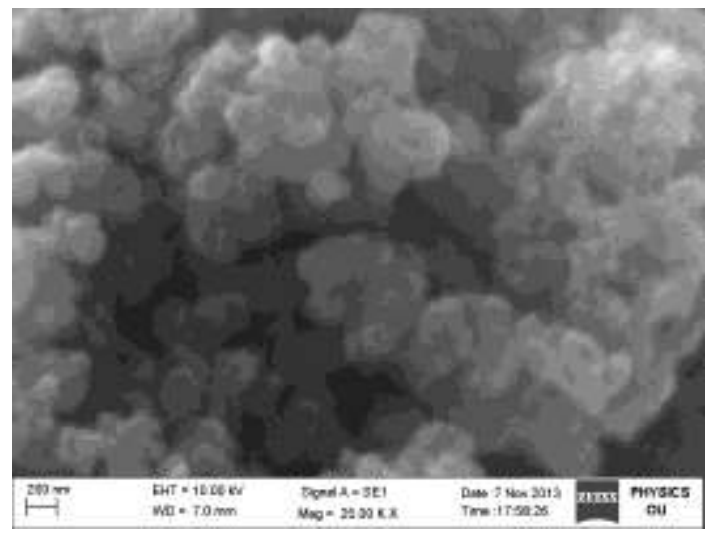

a) PANI

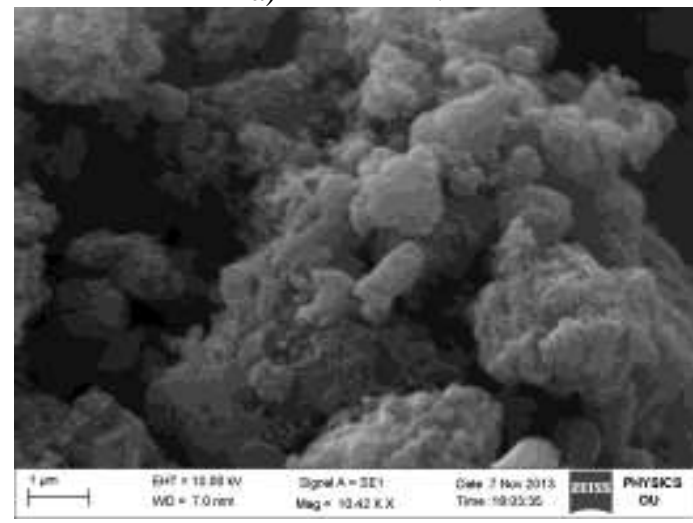

b) PANI/50wt\%Composite

c)

\subsection{SEM image of PANI\& PANI/MoO ${ }_{3}$ Composite}

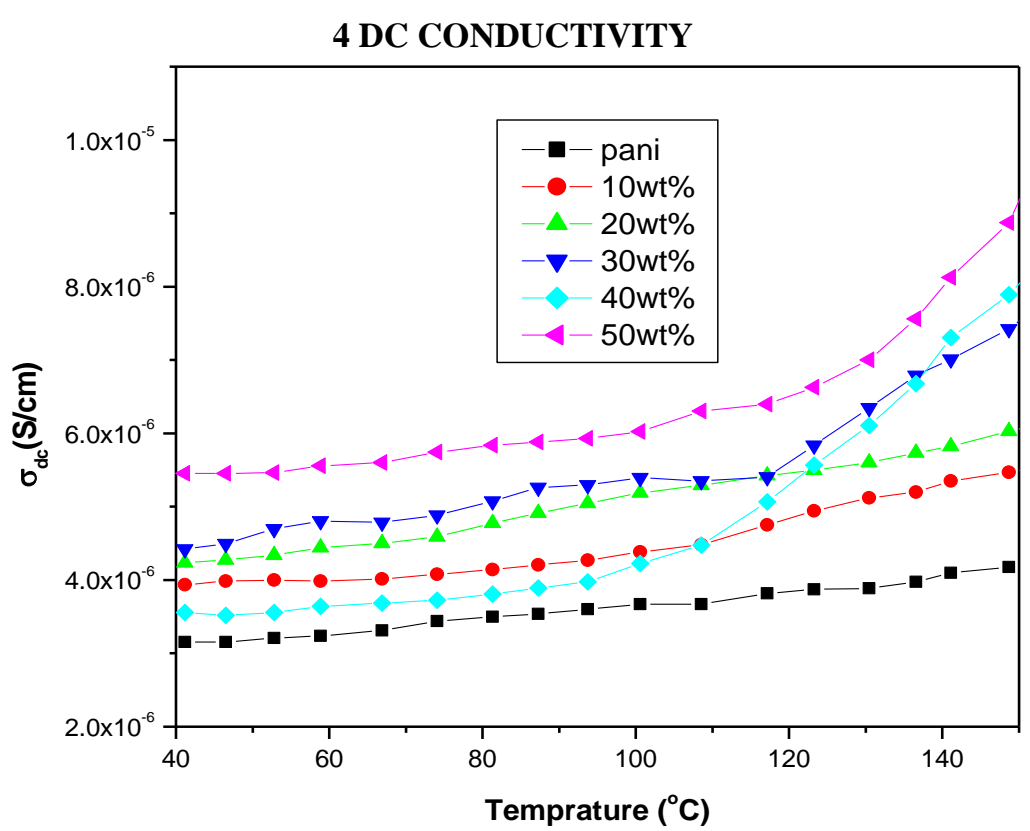

Fig 4 shows the conductivity with temperature for PANI- $\mathrm{MoO}_{3}$ composites

The increase in conductivity for the composites may be due to the formation of more number of polarons the change in the conductivity of the composites indicates a change in the doping state of the polymer. Though the composite having $50 \mathrm{wt} \%$ of PANI/ $\mathrm{MoO}_{3}$ shows the higher conductivity than pure PANI such enhancement of DC conductivity values can be attributed to the uncoiling of polymeric chains due to strong interfacial interaction between $\mathrm{MoO}_{3}$ with PANI caused by their composition . 


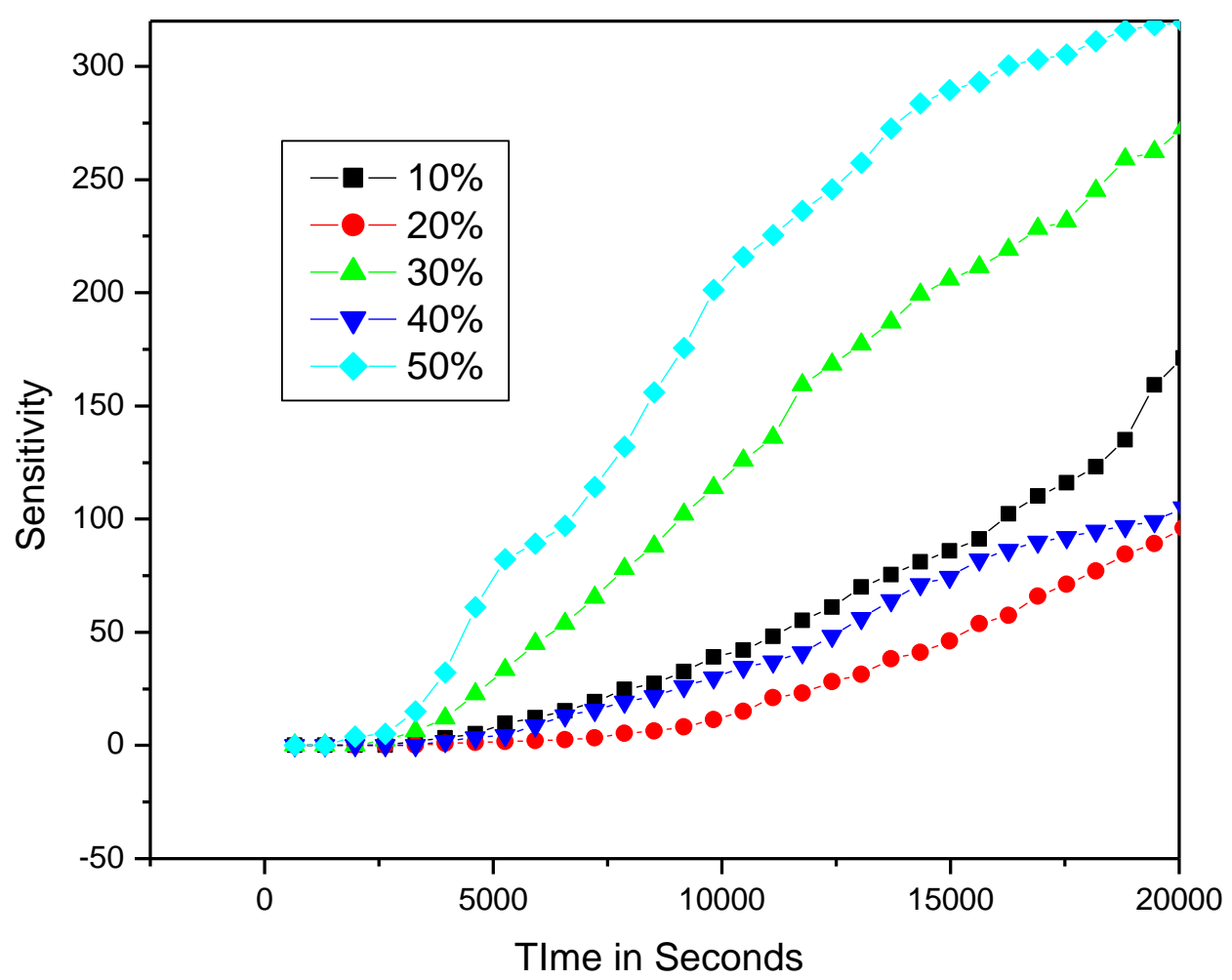

Figure 5. The Sensitivity study of PANI- $\mathrm{MoO}_{3}$ nanocomposite

\section{SENSOR STUDIES}

The increasing sensitivity at higher weight percentage may be due to high surface area with possible reaction sites of the nanocomposites due to adsorption of gas molecules. The gas sensing mechanism of $\mathrm{MoO}_{3}$ based LPG sensor is a surface controlled phenomenon i.e., it is based on the surface area of the nanoparticles at which the LPGmolecules adsorbs and reacts with pre-adsorbed oxygen molecules.It is found that the large specific area contributes to the oxygen and LPG adsorption on the surface of the materials, which is responsible for the increase in sensitivity of the sensor .

\section{CONCLUSION}

The prepared nanocomposites were characterized SEM to study the morphology of the composites. The electrical study indicates here was a strong influence on the conductivity of small dopant added to the conducting polymer. It is observed that conductivity and Sensitivity increases with the increase in the concentration of $\mathrm{MoO}_{3}$ particles. Hence these composites are found to be promising material for potential applications.

[1] K. K.E. George, Acta Materialia, 57, 371-375 (2009)

\section{REFERENCES}

[2] S. K. Dhawan, AIP Advances 1, 022147-11 (2011)

[3] K.H.Wu, Journal of Magnetism and Magnetic Materials 323 (2011) 933-938

[4] Yu, Applied Surface Science 258, 3184-3190 (2012)

[5] Ping Xu, and Xijiang Han, J. Phys. Chem. C, 114, 19600-19606 (2010)

[6] , Physica B, 406, 1950-1955 (2011)

[7] [27] Singh R, Kaur A, Yadav K L and Bhattacharya D, Curr. Appl. Phys, 3 (2006) 235-8

[8] [28] A. Moezzi., A.M. McDonagh., M.B.Cortie..J.ChemEng Journal.1-22,(2012),186.

[9] [29]). A.S.Roy, S.Gupta, S.Seethamraju, G.Madras, P.C.Ramamurthy,Composites: Part B 58, $\quad$ (2014), 139.

[10] [30]. Connolly J and St Pierre T G 2001 J. Magn. Magn. Mater. 225156 